\title{
Does intervention always essential for aorto-right ventricular outflow tract fistula after surgical aortic valve replacement? : A case report
}

\author{
Ibrahim Halil Inanc \\ Department of Cardiology, Besni State Hospital, Adiyaman, Turkey
}

Received: 2020-06-22.

Accepted: 2020-07-16

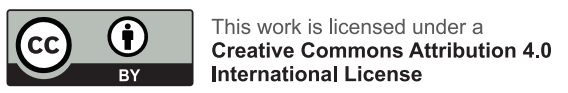

J Clin Med Kaz 2020; 5(59):54-56

Corresponding author:

Dr. Ibrahim Halil INANC.

E-mail: dr.ibrahimhalilinanc@outlook.com

ORCID: https://orcid.org/0000-0003-4046-6748

\section{Abstract}

Surgical aortic valve replacement is an effective treatment for symptomatic severe aortic stenosis in patients with low-intermediate operative risk. Prosthetic valve endocarditis is a complication after aortic valve replacement and accounts for $20 \%$ of infective endocarditis. Prosthetic valve endocarditis sometimes complicates with abscess formation, pseudoaneurysm and aorto-cardiac fistula. Although severity of symptoms mostly depend on shunt's size, aorto-cardiac fistula has high mortality rate despite aggressive surgical and percutaneous intervention. Rarely, small shunts can be tolerated.

We describe a case of aorto-right ventricle outflow tract fistula presented with signs and symptoms of congestive heart failure and remained asymptomatic under medical therapy.

Key words: aortic valve replacement, pseudoaneurysm, aorto-right ventricle outflow tract fistula

\section{Introduction}

Calcific aortic stenosis (AS) is the most common heart valve disease in developed countries [1]. Aortic valve replacement (AVR) is preferred for symptomatic severe AS patients with low-intermediate operative risk [2]. Prosthetic valve endocarditis (PVE) is one of the most dangerous complication after AVR of and accounts for $20 \%$ of all cases of infective endocarditis. It occurs $3 \%$ to $6 \%$ of patients within 5 years after surgery and associated with high mortality and morbidity $[3,4]$. PVE sometimes complicates with invasion and destruction of the periannular tissue resulting in abscess formation. This may lead to aortic perivalvular pseudoaneurysm and more rarely erode into adjacent cardiac chambers, leading to an ACF which has high mortality rate. This condition generally requires urgent cardiac surgery, but patients rarely remain asymptomatic [5].

We present the of case of a 69 -year-old male patient diagnosed with aorta-right ventricle outflow tract (RVOT) fistula 2 months after AVR and remained asymptomatic under medical therapy.

\section{Case-presentation}

69-year-old male patient with a history of surgical AVR because of severe AS referred to our clinic suffering from shortness of breath during exertion.
AVR was performed 2 months ago. He had a history of hemodialysis because of persistent pulmonary edema attacks and renal dysfunction 3 weeks ago. He received intravenous antibiotic treatment for mild respiratory tract infection during that period. On his physical exam, there was a precordial grade 3/6 murmur; prosthetic clicks were heard clearly. Vital signs were; Blood pressure: 110/70 $\mathrm{mmHg}$, Temperature: $37,8^{\circ} \mathrm{C}$, Pulse: 98 bpm, Saturation: $95 \%$ on room air. Clinical laboratory test results revealed an increase of inflammation related markers (Sedimentation Rate: $38 \mathrm{~mm} / \mathrm{hr}$, C-reactive protein: 68 $\mathrm{mg} / \mathrm{L}, \mathrm{WBC}$ : 14.2x109/L). Plasma creatinine level was $2.1 \mathrm{mg} / \mathrm{dl}$ and Glomerular filtration rate (GFR) was 34.5. Transthoracic echocardiography (TTE) revealed non dilated chambers with prosthetic aortic valve-in-situ with peak and mean gradient of 25 and $10 \mathrm{mmHg}$. There was an abnormal communication between aortic root and RVOT starting from aortic annulus level, continuing through aortic wall and draining into the RVOT (Figure 1). The shunt was confirmed from different TTE views with continuous unidirectional color-flow jet (Figure 2,3). There was minimal paravalvular aortic regurgitation but not significant prosthesis dehiscence. RV size was normal and systolic pulmonary artery pressure (sPAP) was 30 mmHg. Qp/Qs was 1.4/ 1.0. 
Figure 1 - Two-dimensional transthoracic echocardiogram parasternal long axis (PLAX) view of fistula tract (arrow)

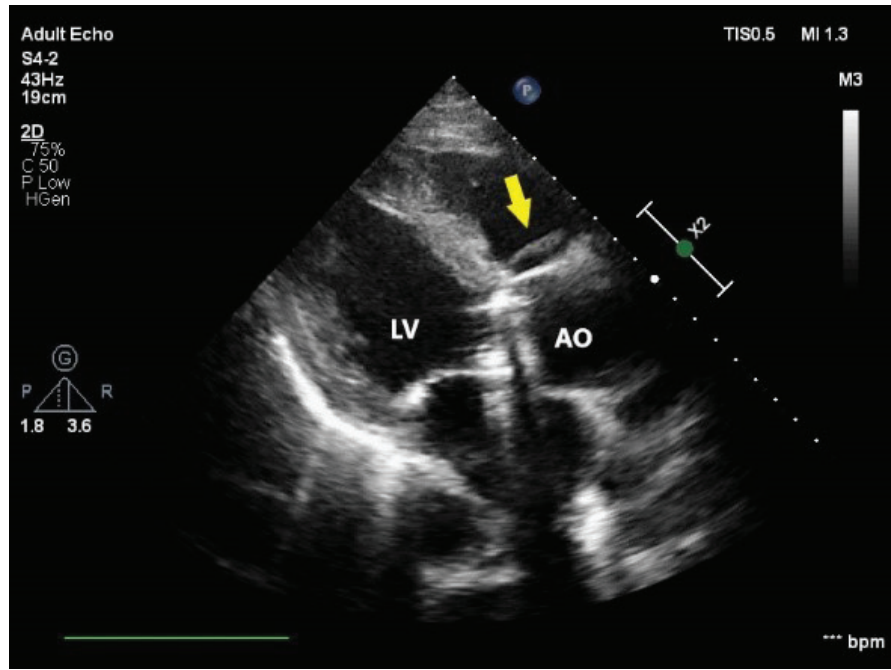

Figure 2 - Two-dimensional transthoracic echocardiogram parasternal long axis (PLAX) view with color flow doppler of fistula tract (arrow)

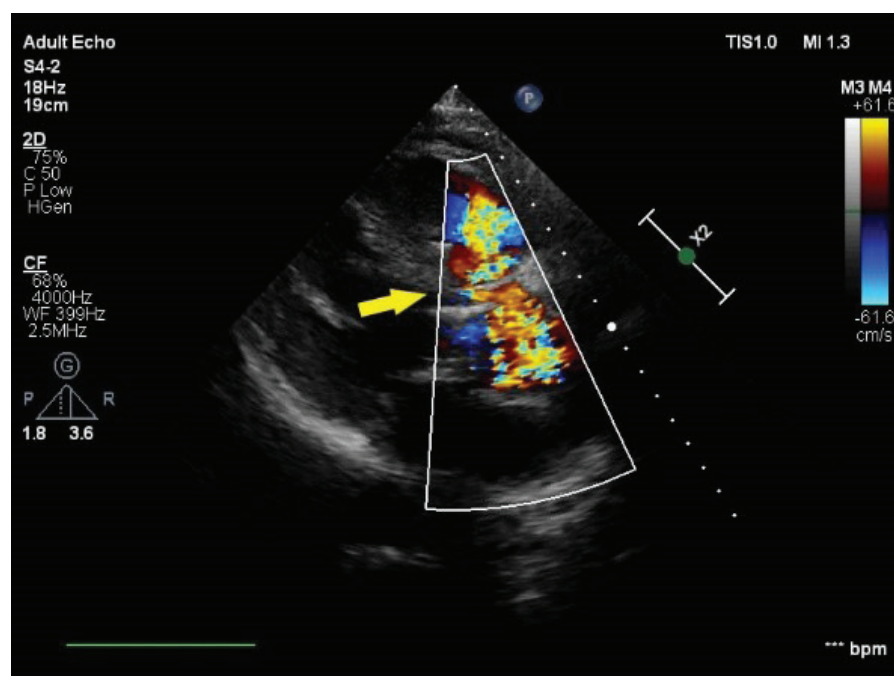

Figure 3 - Two-dimensional transthoracic modified subcostal view with color flow doppler of fistula tract (arrow)

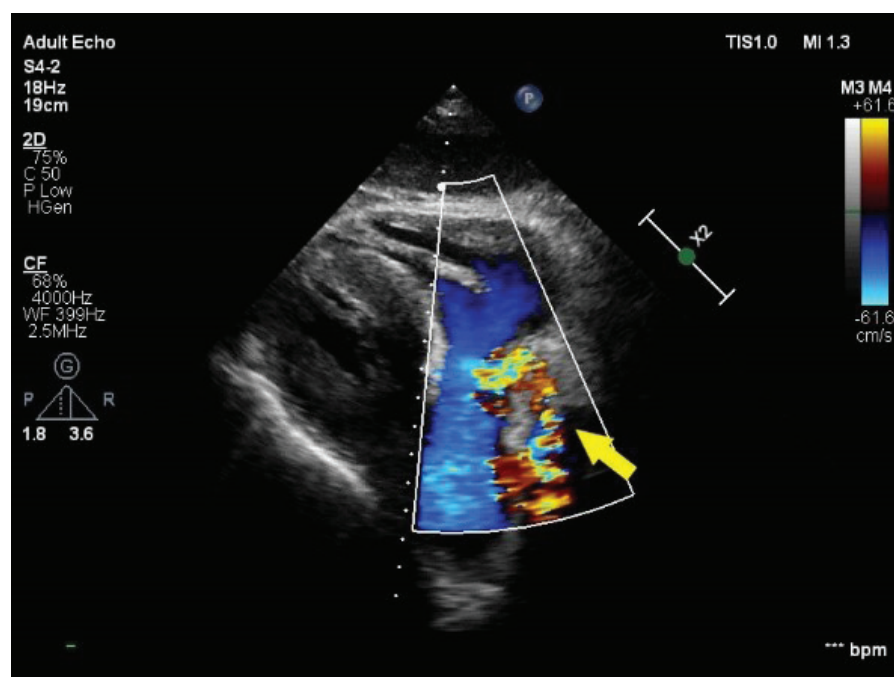

Transesophageal echocardiography (TEE) also confirmed $3 \times 20 \mathrm{~mm}$ fistula tract from different views (Figure 4,5).
Figure 4 - Midesophageal long axis view with color flow doppler of fistula from aortic root to right ventricle outflow tract (RVOT)

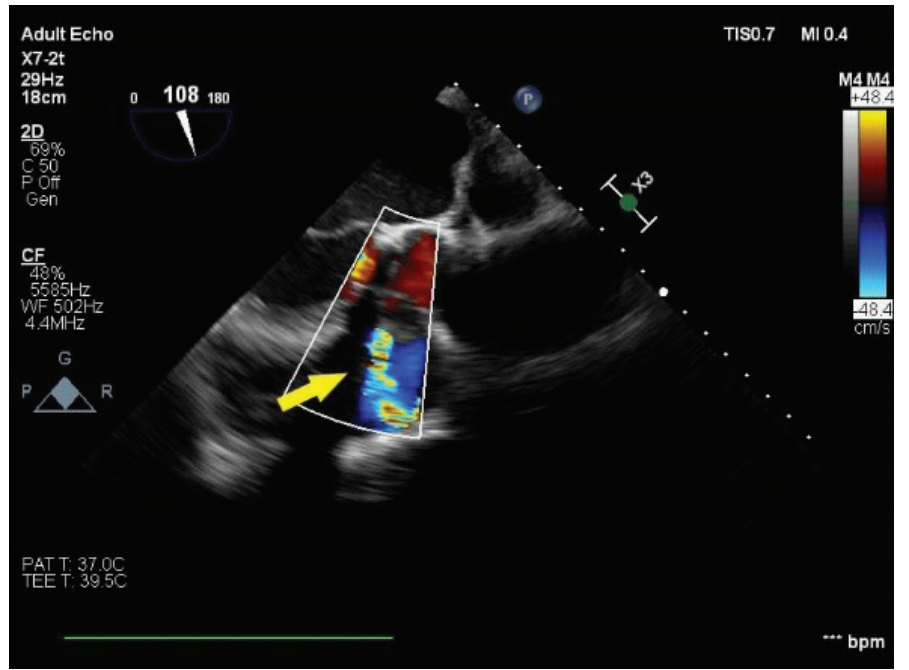

Figure 5 - Two-dimensional transthoracic echocardiogram parasternal long axis (PLAX) view with color flow doppler of fistula tract (arrow)

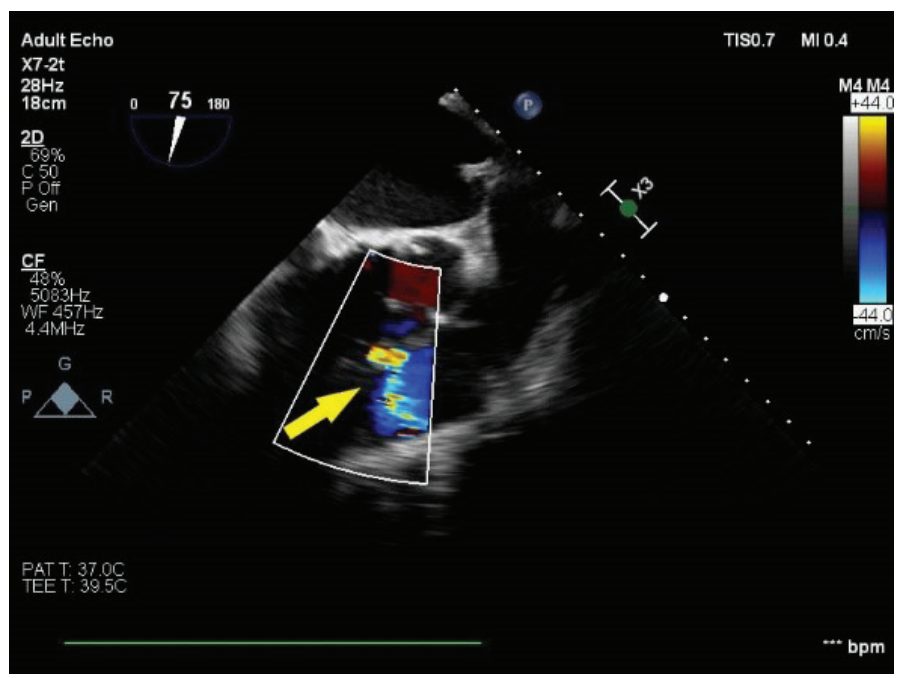

Patient refused re-operation and was hospitalized for antibiotic treatment for 6 weeks because of infective endocarditis. None of blood culture results were positive. During clinical and echocardiographic follow up under medical therapy for 8 months, he had no sign of hypervolemia.

\section{Discussion}

Aorto-cardiac fistulas (ACF) are rare but more frequently reported and data is limited. The causes of ACFs are mostly iatrogenic, infectious and traumatic. In our patient, PVE most probably led to aortic perivalvular abscess formation, pseudoaneurysm and aorta-right ventricule outflow track fistula. Fistulas are mostly located between aorta-right atrium and aorta-pulmonary artery. Aorta-right ventricular or aorta-right ventricular outflow tract fistulas are less common.

Patients may present with sudden clinical deterioration and congestive heart failure symptoms. Some patients suffer from exertional dyspnea and right heart failure symptoms such as ascites and peripheral edema. The severity of symptoms mostly depends on shunt's size. Although small shunts can be tolerated, majority of cases undergo reoperation [6]. Rarely, symptomatic patients can be treated with percutaneously. For asymptomatic 
patients who are diagnosed on an imaging study, medical treatment can be an option; but patients being treated without any intervention have significantly higher mortality rates $[7,8]$. Our patient was not suitable percutaneous closure because of the curves, shape and course of fistula tract. Reoperation was planned but he refused.

The answer of question why patient had recurrent pulmonary edema attacks despite a small shunt was probably underlying kidney dysfunction and acute PVE contributing clinical deterioration. After that period he remained asymptomatic during antibiotheraphy and follow-up.

Although ACF is rare, it is one of the most serious complication of IE and has a high mortality rate despite aggressive treatment with surgical intervention in the majority of patients [9] After acute episode, the patient might tolerate if volume of the shunt is not excess. TTE with color-flow Doppler, TEE or both may be required for diagnosis. Careful follow-up of these patients is essential because heart failure may develop in some patients any time, necessitating surgical correction of the fistula.

Disclosures: There is no conflict of interest for all authors.

Funding: The authors declared that this study has received no financial support.

\section{References}

1. Lindman BR, Clavel M-A, Mathieu P, Iung B, Lancellotti P, Otto CM, et al. Calcific aortic stenosis. Nature reviews Disease primers. 2016; 2(1):1-28. https://doi.org/10.1038/nrdp.2016.6

2. Razzolini R, Covolo E. Severe Aortic Stenosis Treatment: Percutaneous Options, Patient Selection, and Preoperative Evaluation. Percutaneous Interventions for Structural Heart Disease: Springer. 2017; 3-13. https://doi.org/10.1007/978-3-319-43757-6_1

3. Glaser N, Jackson V, Holzmann MJ, Franco-Cereceda A, Sartipy U. Prosthetic valve endocarditis after surgical aortic valve replacement. Circulation. 2017; 136(3):329-31. https://doi.org/10.1161/CIRCULATIONAHA.117.028783

4. Lalani T, Chu VH, Park LP, Cecchi E, Corey GR, Durante-Mangoni E, et al. In-hospital and 1-year mortality in patients undergoing early surgery for prosthetic valve endocarditis. JAMA internal medicine. 2013; 173(16):1495-504. https://doi.org/10.1001/ jamainternmed.2013.8203

5. Habib G, Lancellotti P, Antunes MJ, Bongiorni MG, Casalta J-P, Del Zotti F, et al. 2015 ESC guidelines for the management of infective endocarditis: the task force for the management of infective endocarditis of the European Society of Cardiology (ESC) endorsed by: European Association for Cardio-Thoracic Surgery (EACTS), the European Association of Nuclear Medicine (EANM). European heart journal. 2015; 36(44):3075-128. https://doi.org/10.1093/eurheartj/ehv319

6. Konda MK, Kalavakunta JK, Pratt JW, Martin D, Gupta V. Aorto-right ventricular fistula following percutaneous transcatheter aortic valve replacement: case report and literature review. Heart views: the official journal of the Gulf Heart Association. 2017 ; $18(4): 133$. https://doi.org/10.4103/HEARTVIEWS.HEARTVIEWS_115_16

7. Foster TJ, Amin AH, Busu T, Patel K, Farjo P, Al Hallak A, et al. Aorto-cardiac fistula etiology, presentation, and management: A systematic review. Heart \& Lung. 2019; https://doi.org/10.1016/j.hrtlng.2019.11.002

8. Al-Maskari S, Panduranga P, Al-Farqani A, Thomas E, Velliath J. Percutaneous closure of complex paravalvular aortic root pseudoaneurysm and aorta-cavitary fistulas. Indian heart journal. 2014; 66(3):358-62. https://doi.org/10.1016/j.ihj.2014.03.015

9. Anguera I, Miro JM, San Roman JA, de Alarcon A, Anguita M, Almirante B, et al. Periannular complications in infective endocarditis involving prosthetic aortic valves. The American journal of cardiology. 2006; 98(9):1261-8. https://doi.org/10.1016/j. amjcard.2006.05.066 